DOI: 10.17516/1999-494X-0353

УДК 621.314: 621.43:536.2

\title{
Numerical Study of the Influence \\ of the Characteristics of Fuel Injectors \\ on the Ecological Parameters of a Diesel-Generator
}

\author{
Dmitriy V. Guzei ${ }^{\mathrm{a}}$, Vasiliy I. Panteleeva, \\ Andrey V. Minakov ${ }^{\mathrm{a}, \mathrm{b}}$ and Vladimir A. Zhigarev*a \\ ${ }^{a}$ Siberian Federal University \\ Krasnoyarsk, Russian Federation \\ ${ }^{b}$ Kutateladze Institute of Thermophysics SB RAS \\ Novosibirsk, Russian Federation
}

Received 22.07.2021, received in revised form 16.08.2021, accepted 21.09.2021

\begin{abstract}
The paper presents the results of a systematic computational study to optimize the operation of diesel generator fuel injectors. The influence of the diameter of the outlet nozzle of the nozzle and the inclination of its axis, the taper angle of the spray and the injection advance angle on the local and integral characteristics of the operation of the diesel generator, as well as the values of emissions of soot, nitrogen oxides and underburning of fuel, has been investigated. It is shown that even with a slight optimization of the performance of the fuel injectors, it is possible to significantly reduce harmful emissions during the operation of a diesel generator.
\end{abstract}

Keywords: diesel-generator sets, variable speed, fuel injectors, ignition, optimization, emissions of harmful substances, mathematical model.

Citation: Guzei D. V., Panteleev V. I., Minakov A. V., Zhigarev V. A. Numerical study of the influence of the characteristics of fuel injectors on the ecological parameters of a diesel-generator, J. Sib. Fed. Univ. Eng. \& Technol., 2021, 14(6), 667-683. DOI: $10.17516 / 1999-494 X-0353$

(C) Siberian Federal University. All rights reserved

This work is licensed under a Creative Commons Attribution-Non Commercial 4.0 International License (CC BY-NC 4.0).

* Corresponding author E-mail address: VZhigarev@sfu-kras.ru 


\title{
Расчетное исследование влияния характеристик топливных форсунок на экологические параметры дизель-генератора
}

\author{
Д.В. Гузей ${ }^{\mathrm{a}}$ В.И. Пантелеев ${ }^{\mathrm{a}}$, \\ А. В. Минаков ${ }^{\text {a, }}$, В.А. Жигарев ${ }^{a}$ \\ ${ }^{a}$ Сибирский федеральный университет \\ Российская Федерация, Красноярск \\ ${ }^{6}$ Институт теплофизики им. С. С. Кутателадзе СО РАН \\ Российская Федерация, Новосибирск
}

\begin{abstract}
Аннотация. В работе представлены результаты систематического расчетного исследования по оптимизации работы топливных форсунок дизель-генератора. Исследовано влияние диаметра выходного сопла форсунки, наклона ее оси, угла конусности спрея и угла опережения впрыска на локальные и интегральные характеристики работы дизель-генератора, а также количество выбросов сажи, окислов азота и недожога топлива. Показано, что даже при незначительной оптимизации характеристик работы топливных форсунок можно в значительной мере добиться снижения вредных выбросов в процессе работы дизель-генератора.
\end{abstract}

Ключевые слова: дизель-генераторные установки, переменная частота вращения, топливные форсунки, угол опережения зажигания, оптимизация, выбросы вредных веществ, математическая модель.

Цитирование: Гузей, Д. В. Расчетное исследование влияния характеристик топливных форсунок на экологические параметры дизель-генератора / Д. В. Гузей, В. И. Пантелеев, А. В. Минаков, В. А. Жигарев // Журн. Сиб. федер. ун-та. Техника и технологии, 2021, 14(6). С. 667-683. DOI: 10.17516/1999-494X-0353

\section{Введение}

Дизель-генераторные установки (ДГУ) традиционно используются в качестве локальных источников генерации электроэнергии. Особенно широкое распространение ДГУ получили для электроснабжения удаленных и изолированных арктических поселков. Чаще всего такие источники генерации большую часть времени работают на нагрузку меньше номинальной, что приводит к завышенному потреблению дизельного топлива и к сокращению ресурса работы дизельного двигателя. Кроме того, подавляющее большинство дизель-генераторов работает со стабильной скоростью вращения вала дизеля независимо от мощности нагрузки. Такой режим работы дизеля также является неоптимальным с точки зрения потребления топлива. Кроме того, в последнее время из-за ввода все более жестких природоохранных ограничений важнейшую роль приобретают экологические параметры работы двигателей внутреннего сгорания. Основные вредные выбросы от работы дизельных двигателей - сажа, оксиды азота и оксиды серы, а также продукты неполного сгорания топлива. Для экономии дизельного топлива и продления ресурса двигателя и снижения вредных выбросов предлагается регулировать его частоту вращения вала в функции нагрузки электрической сети [1-4]. Однако при этом остаётся необходимость обеспечения стабильных значений напряжения и частоты в электрической сети, питающей потребителей электроэнергии. Этим и другим требованиям в большей степени соответствуют разработанные в последнее время дизель-генераторные установки с переменной 
частотой вращения. Это новый тип генерирующего оборудования с применением вентильноиндукторных генераторов, имеющего ряд преимуществ перед широко используемыми сегодня ДГУ с синхронными генераторами.

Для дальнейшего совершенствования параметров работы дизель-генераторных установок в нашей недавней работе [5] представлена комплексная математическая модель процессов тепломассообмена в камере сгорания дизельного двигателя. В отличие от известных ранее моделей, основанных на решении систем обыкновенных дифференциальных уравнений, данная математическая модель основана на методах вычислительной гидродинамики (CFD). Модель учитывает реальную геометрию камеры сгорания и условия работы дизельного двигателя, характеристики работы форсунок и свойства дизельного топлива. С помощью разработанной модели проведено исследование основных характеристик дизель-генератора в широком диапазоне режимов его работы. Проанализированы характеристики распыла и воспламенения топлива в камере сгорания. Исследованы особенности поведения локальных и интегральных характеристик камеры сгорания двигателя.

Целью настоящего исследования является систематическое расчетное исследование и оптимизация характеристик топливных форсунок дизельного двигателя для снижения вредных выбросов. Несмотря на то что в последнее время этому вопросу уделяется повышенное внимание [6-8], окончательной ясности здесь еще нет. Это требует дальнейших исследований, выполненных в данной работе.

\section{1. Математическая модель и постановка моделирования}

Для моделирования процессов, происходящих в камере сгорания двигателя дизельгенератора с переменной частотой вращения, была разработана математическая модель процессов горения в камере сгорания с учетом характеристик впрыска, воспламенения и сгорания топливной смеси. Детально модель была описана в нашей работе [5]. Здесь же отметим основные моменты.

Математическая модель процессов тепломассообмена в камере сгорания двигателя состоит из следующих основных уравнений, основанных на законах сохранения.

Уравнение сохранения массы газовой смеси:

$$
\frac{\partial \bar{\rho}}{\partial t}+\nabla \cdot(\bar{\rho} \widetilde{\boldsymbol{u}})=\dot{\bar{\rho}}^{s}
$$

Здесь $\widetilde{\boldsymbol{u}}$ - вектор скорости потока, $\bar{\rho}$ - плотность смеси, $\dot{\bar{\rho}}_{k}^{s}-$ источник массы в процессе испарения дизельного топлива.

Уравнение сохранения импульса смеси учитывает свободную конвекцию, силы давления, силы вязкого трения, турбулентные напряжения, а также импульс, вносимый при распыливании спрея дизельного топлива в камере сгорания:

$$
\frac{\partial \bar{\rho} \widetilde{\boldsymbol{u}}}{\partial t}+\nabla \cdot(\bar{\rho} \widetilde{\boldsymbol{u}} \widetilde{\boldsymbol{u}})=-\nabla \bar{p}+\nabla \cdot \overline{\boldsymbol{\sigma}}-\nabla \cdot \Gamma+\overline{\mathbf{F}}^{s}+\bar{\rho} \overline{\mathbf{g}},
$$

где $\bar{p}$ - давление; $\bar{F}^{s}$ - сила, вносимая в единицу объема при распылении топлива; $\overline{\mathbf{g}}$-ускорение свободного падения; $\Gamma=\bar{\rho}(\widetilde{\boldsymbol{u}} \widetilde{\boldsymbol{u}}-\widetilde{\boldsymbol{u}} \widetilde{\boldsymbol{u}})-$ тензор рейнольдсовых напряжений, который в рамках RANS подхода определяется согласно гипотезе Буссинеска. Для моделирования турбулентности при этом использовалась модель нестационарная $\mathrm{k}-\varepsilon$ RANS модель для нормализованных

$$
-669-
$$


групп (RNG) с дополнительными членами, учитывающими взаимодействие турбулентных вихрей и капель спрея [9].

Уравнение сохранения энергии учитывает работу сил давления, конвекцию и теплопроводность, турбулентную диссипацию энергии, тепловыделение в процессе химических реакций и испарения топлива и записывается для внутренней энергии в следующем виде:

$$
\frac{\partial \bar{\rho} \tilde{\mathbf{I}}}{\partial t}+\nabla \cdot(\bar{\rho} \tilde{\boldsymbol{u}} \tilde{\mathbf{I}})=-\bar{p} \nabla \cdot \widetilde{\boldsymbol{u}}-\nabla \cdot \overline{\mathbf{J}}-\nabla \cdot \mathbf{H}+\bar{\rho} \tilde{\varepsilon}+\dot{\bar{Q}}^{C}+\dot{\bar{Q}}^{s},
$$

где I - удельная внутренняя энергия; $\overline{\mathbf{J}}$ - вектор теплового потока; $\dot{\bar{Q}}^{c}$ и $\dot{\bar{Q}}^{S}$ - источниковые члены, связанные с тепловыделением в процессе горения и испарения топлива соответственно; Н - турбулентный поток тепла.

Для моделирования распыливания дизельного топлива использована модель полного конуса, которая включает в себя распыливание топлива, дробление его капель, их столкновение и слияние, испарение. При этом начальные характеристики спрея (диаметр капель и их скорость) определяли эмпирически с помощью коэффициента расхода форсунки. Для моделирования дальнейшего распада и дробления капель топлива использовали модель Рэлея-Тейлора [10]. Для моделирования испарения капель дизельного топлива применена многокомпонентная модель. Данная модель испарения рассматривает сферическую каплю жидкости, которая состоит из конечного числа компонентов, испаряющихся без химических реакций в газовой среде [11]. При этом дизельное топливо представлялось моделью суррогатного топлива - n-гептан $\left(\mathrm{nC}_{7} \mathrm{H}_{16}\right)$. Для моделирования кинетики горения дизельного топлива в газовой фазе использовался кинетический механизм (Diesel_1COMp_35sp_CHeM.iNp), содержащий 74 элементарные реакции и 35 газовых компонент. Для моделирования влияния турбулентности на кинетику горения топлива была взята обобщенная модель взаимодействия турбулентности и химии из работы Kong et al. [12], которая широко применяется для моделирования дизельных двигателей. Эта модель предполагает, что скорость химической реакции частично ограничивается скоростью турбулентного перемешивания. Для моделирования образования сажи в камере сгорания дизельного двигателя использовали двухстадийную модель, состоящую из конкурирующих механизмов образования [13] и окисления [14] частиц сажи. Особенностью моделирования процессов в камере сгорания двигателя является необходимость учитывать изменение объема расчетной области в процессе движения поршня. В данной работе для расчета процесса движения поршня использован лагранжев метод динамических сеток (ALE). В процессе движения поршня расчетные ячейки деформируются вместе с уменьшением объема камеры сгорания. Процесс сжатия и расширения газов описывается уравнениями состояния для смеси идеальных газов. Задача является существенно нестационарной.

Дискретизация уравнений переноса осуществлялась по методу контрольного объема на неструктурированной сетке. Связь полей скорости и давления для несжимаемой жидкости реализовывалась с помощью процедуры SIMPLE. Для аппроксимации конвективных членов уравнения на компоненты импульса была взята противопоточная схема второго порядка QSOU. Для аппроксимации конвективных членов уравнения на турбулентные характеристики использовалась противопоточная схема второго порядка. Нестационарные слагаемые аппроксимировались по неявной схеме 1-го порядка точности. Диффузионные члены аппроксимировались по схеме 2-го порядка. 
Исследование проведено для широко применяемого в дизель-генераторных установках дизельного двигателя в широком диапазоне частоты вращения коленчатого вала. Был рассмотрен рядный шестицилиндровый дизельный двигатель. Рабочий объем всех цилиндров 6.65 л. Диаметр цилиндра равен 105 мм, ход поршня 128 мм, длина шатуна 320 мм. Степень сжатия 17.5. Частота вращения коленчатого вала при номинальной мощности 2300 об/мин. Форсунка закрытого типа с многоструйным распылением, количество струй 7 , диаметр сопла 0.182 мм. Максимальное давление впрыска, обеспечиваемое топливной системой, 180 МПа. Температура окружающей среды в расчетах была равна $293 \mathrm{~K}$. Температура свежего заряда с учетом охлаждения воздуха в охладители принята равной 399 К. Средняя температура стенок была равна $450 \mathrm{~K}$.

Построена пространственная геометрия расчетной области, представляющая внутреннюю часть камеры сгорания дизельного двигателя. Поскольку геометрия осесимметрична, с целью сокращения расчетного времени был взят сектор в $30^{\circ}$ (рис. 1a). Для расчетов использовалась многоблочная структурированная криволинейная сетка, состоящая из 200000 расчетных ячеек (рис. 1б).

В работе [5] с помощью разработанной модели проведено исследование основных характеристик дизель-генератора в широком диапазоне режимов его работы. Частота вращения коленчатого вала в расчетах варьировалась от 900 до 2400 об/мин. Проанализированы характеристики распыла, дробления и воспламенения топлива в камере сгорания. Исследованы особенности поведения локальных и интегральных характеристик камеры сгорания двигате-

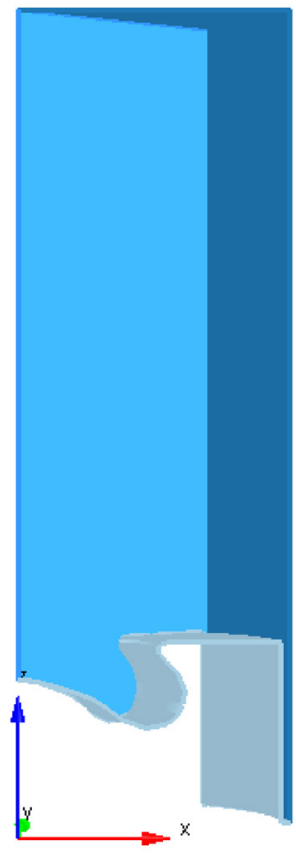

a)

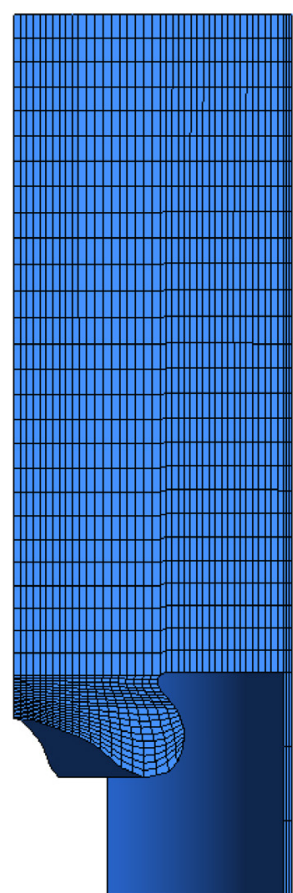

б)

Рис. 1. Геометрия расчетной области $(a)$ и расчетная сетка (б)

Fig. 1. The geometry of the computational domain (a) and the computational grid ( $\sigma)$

$$
-671-
$$


ля. При этом рассмотрены не только его энергетические характеристики, но и экологические параметры. На рис. 2 в качестве примера показано поведение в процессе движения поршня осредненных по объему цилиндра температуры, объемной доли частиц сажи, окислов азота и недожога топлива для трех различных режимов работы двигателя. Показано, что от режима работы дизель-генератора значительно зависят его экологические характеристики. В режимах, далеких от оптимальных, существенно возрастают выбросы вредных веществ. Так, в частности, было показано, что локальные значения выбросов сажи при увеличении частоты вращения коленчатого вала с 1000 до 2300 об/мин снижаются более чем в 2 раза, локальные значения окислов азота - в 1,7 раза, недожег высокомолекулярных углеводородов - на 30 \%. При этом разброс локальных значений температуры в камере сгорания не превышает 100 градусов.

Однако при увеличении оборотов коленчатого вала возрастает и количество подаваемого в камеру сгорания топлива и воздушной смеси. Поэтому, несмотря на то, что локальные значения вредных выбросов в режимах с пониженной частотой вращения могут быть ниже, валовые выбросы с увеличением частоты вращения, как правило, возрастают. Таким образом, при совершенствовании дизель-генераторов с переменной частотой вращения необходимо

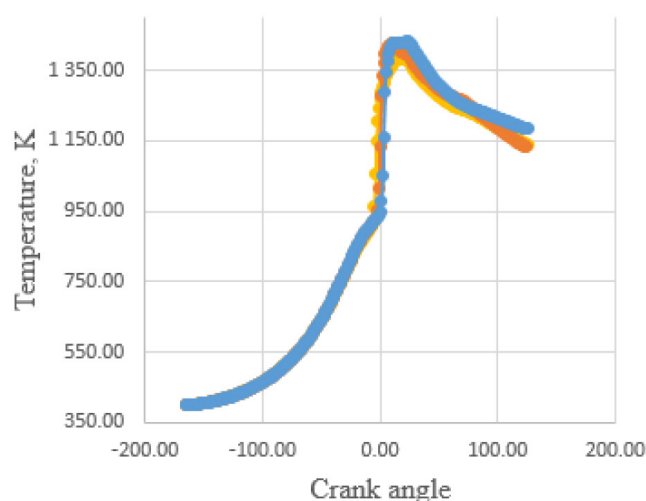

a)

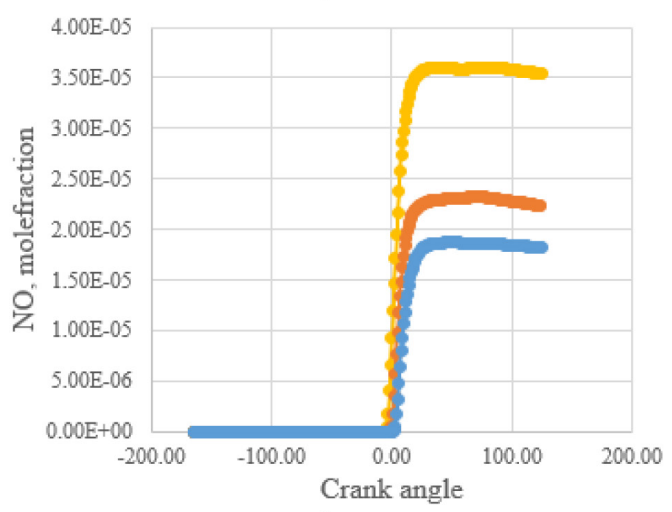

в)

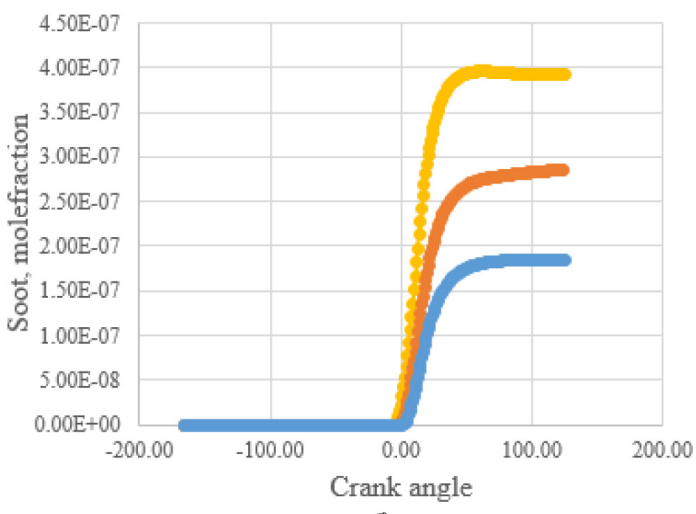

б)

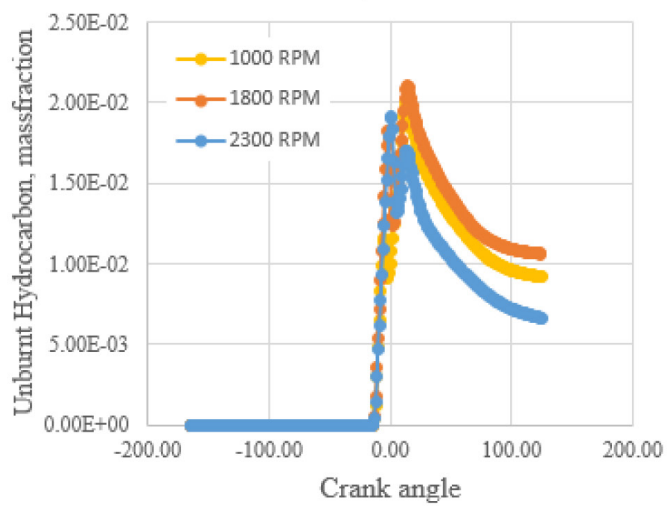

2)

Рис. 2. Средние значения температуры (a), объемной доли сажи (б), окислов азота (в) и недожега топлива (2) в камере сгорания в зависимости от угла поворота коленчатого вала

Fig. 2. Average values of temperature $(a)$, volume fraction of soot $(\sigma)$, nitrogen oxides $(b)$ and underburnt hydrocarbon (2) in the combustion chamber at different rotational speed of the crankshaft 
учитывать множество факторов, нелинейно связанных между собой, включая и экологические характеристики. С помощью разработанной математической модели такая оптимизация была проведена. В качестве наиболее доступного метода управления работой дизельного двигателя, не требующего дорогостоящих капиталовложений, была предпринята попытка оптимизации работы топливных форсунок.

\section{2. Расчетное исследование по оптимизации работы форсунок и характеристик их распыла для дизель-генератора}

В данной работе проведено систематическое расчетное исследование по оптимизации работы форсунок и характеристик их распыла для дизель-генератора. Исследовано влияние диаметра сопла форсунки и ее наклона, угла раскрытия конуса распыла, угла опережения впрыска на локальные и интегральные характеристики работы дизель-генератора. Особое внимание было уделено влиянию характеристик распыла топлива на выбросы в процессе работы дизельгенератора.

\section{1. Исследование влияния диаметра топливных форсунок}

Вначале было рассмотрено влияние диаметра сопла форсунки на локальные и интегральные характеристики работы двигателя. Диаметр сопла является важным параметром форсунки, во многом определяющим качество распыливания топлива. Как показывают опытные данные, при уменьшении диаметра и увеличении числа сопел при прочих равных условиях качество распыливания улучшается. Диаметр сопла форсунки варьировался в широких пределах - от 0,1456 до 0,2275 мм. При этом использовалось приближение, согласно которому остальные характеристики форсунки и двигателя оставались неизменными. Качественно влияние диаметра сопла форсунки на характеристики процессов горения спрея можно видеть на рис. 3-6. Анализ результатов моделирования позволил выделить следующие закономерности. С уменьшением диаметра сопла уменьшается средний диаметр капель, что способствует их более быстрому испарению и воспламенению. С другой

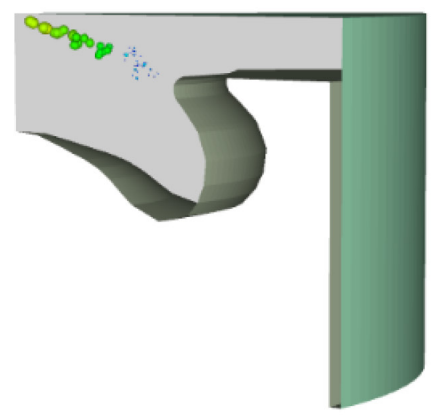

a)

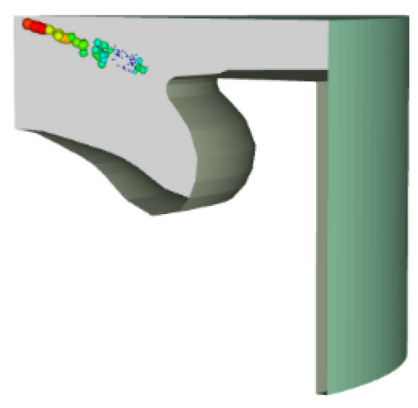

б)

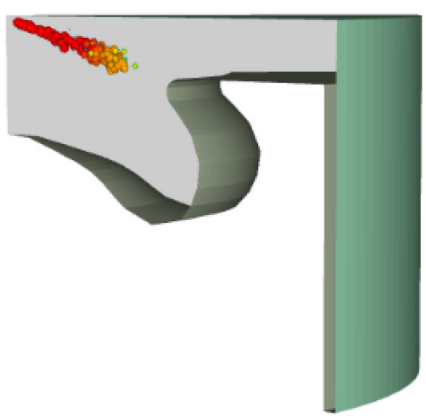

в)

Рис. 3. Характеристики распыла дизельного топлива в камере сгорания при различных диаметрах сопла топливной форсунки: $a-\mathrm{d}=0.1465$ мм; $\sigma-\mathrm{d}=0.182$ мм; $6-\mathrm{d}=0.2275$ мм

Fig. 3. Characteristics of diesel fuel spray in the combustion chamber with different diameters of the fuel injector nozzle: $a-\mathrm{d}=0.1465 \mathrm{~mm}, \sigma-\mathrm{d}=0.182 \mathrm{~mm}, 6-\mathrm{d}=0.2275 \mathrm{~mm}$ 
стороны, с увеличением диаметра капель увеличивается дальнобойность спрея и время пребывания капель в камере сгорания, что также сказывается на характеристиках процесса горения. Более растянутый во времени процесс перемешивания и, следовательно, более длительное время пребывания капель позволяют более богатым смесям воспламениться, делая работу дизель-генератора более устойчивой. Установлено, что с уменьшением диаметра сопла происходит более быстрое перемешивание топлива и окислителя. Это приводит к тому, что быстрее достигается концентрация горючих веществ, необходимая для их воспламенения. Таким образом, из-за более высокой скорости воспламенения и скорости

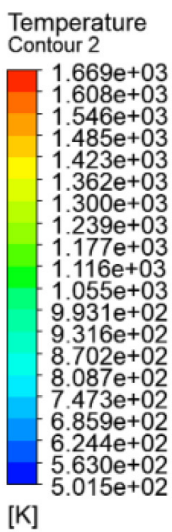
[K]

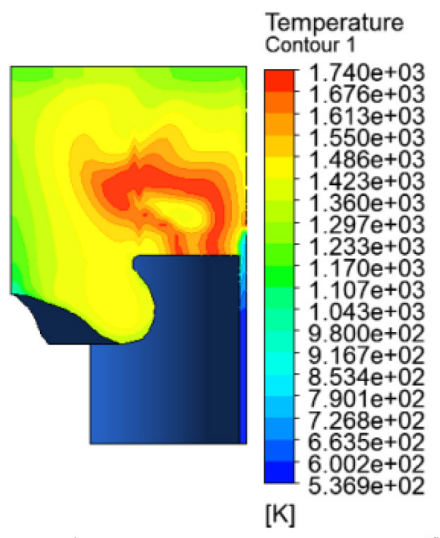

a)
6)

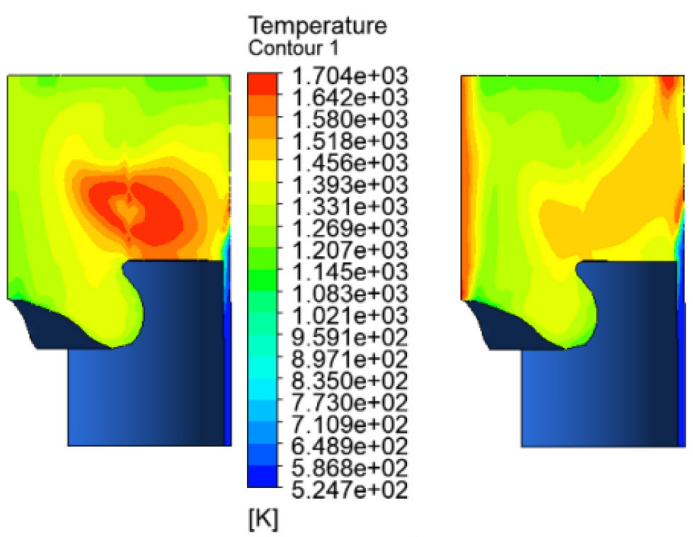

B)

Рис. 4. Температура в камере сгорания дизель-генератора при различных диаметрах сопла топливной форсунки: $a-\mathrm{d}=0.1465$ мм; $\sigma-\mathrm{d}=0.182 \mathrm{мм} ; \boldsymbol{\theta}-\mathrm{d}=0.2275$ мм

Fig. 4. Temperature in the combustion chamber of a diesel generator with different diameters of the fuel injector nozzle: $a-\mathrm{d}=0.1465 \mathrm{~mm}, \sigma-\mathrm{d}=0.182 \mathrm{~mm}, 6-\mathrm{d}=0.2275 \mathrm{~mm}$

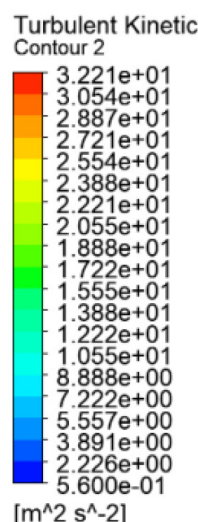

$\left[m^{\wedge} 2 s^{\wedge}-2\right]$

\section{Turbulent Kinetic Energy
Contour 1}

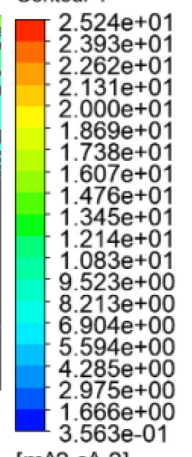

$\left[m^{\wedge} 2 s^{\wedge}-2\right]$

a)

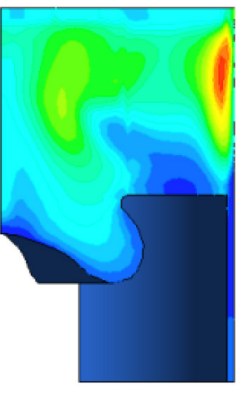

Turbulent Kinetic Energy
Contour 1

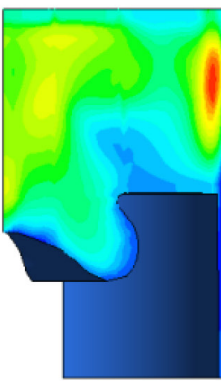

$2.326 \mathrm{e}+01$
$2.204 \mathrm{e}+01$

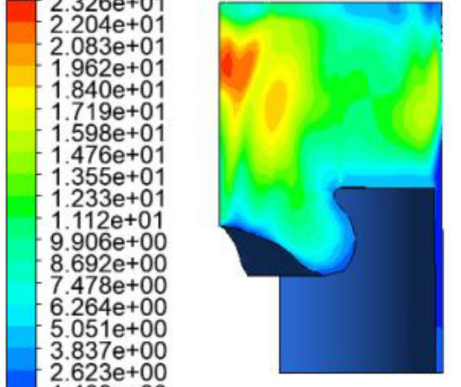

б)
B)

Рис. 5. Турбулентная кинетическая энергия в камере сгорания дизель-генератора при различных диаметрах сопла топливной форсунки: $a-\mathrm{d}=0.1465$ мм; $\sigma-\mathrm{d}=0.182 \mathrm{мм} ; \boldsymbol{c}-\mathrm{d}=0.2275$ мм

Fig. 5. Turbulent kinetic energy in the combustion chamber of a diesel generator with different diameters of the fuel injector nozzle: $a-\mathrm{d}=0.1465 \mathrm{~mm}, \sigma-\mathrm{d}=0.182 \mathrm{~mm}, 6-\mathrm{d}=0.2275 \mathrm{~mm}$ 


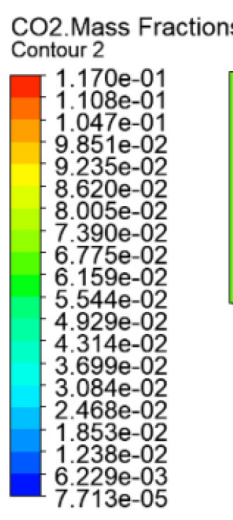

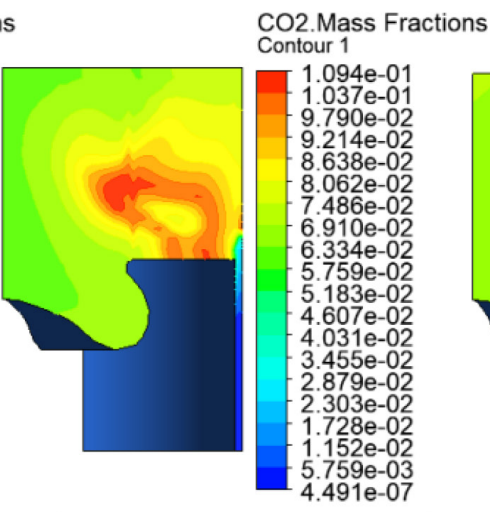

a)

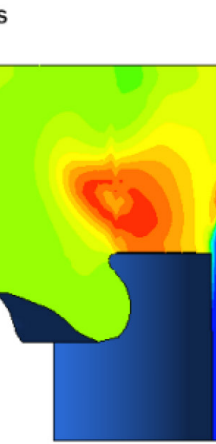

б)
CO2.Mass Fractions Contour 1

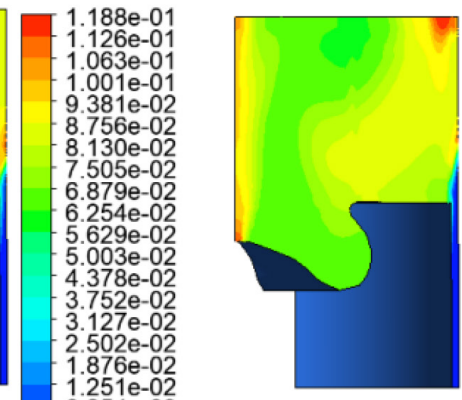

B)

Рис. 6. Распределение массовой доли диоксида углерода в камере сгорания дизель-генератора при различных диаметрах сопла топливной форсунки: $a-\mathrm{d}=0.1465$ мм; $\sigma-\mathrm{d}=0.182 \mathrm{mм} ; в-\mathrm{d}=0.2275$ мм

Fig. 6. Mass fraction $\mathrm{CO}_{2}$ in the combustion chamber of a diesel generator with different diameters of the fuel injector nozzle: $a-\mathrm{d}=0.1465 \mathrm{~mm}, \sigma-\mathrm{d}=0.182 \mathrm{~mm}, 6-\mathrm{d}=0.2275 \mathrm{~mm}$

диссипации с уменьшения сопла форсунки наблюдается смещение фронта пламени по направлению к форсунке.

Описанные выше процессы, связанные с изменением диаметра сопла форсунки, сказываются и на осредненных по объему камеры сгорания характеристиках, приведенных на рис. 7. Как видно, с увеличением диаметра капель существенно снижается максимум температуры к камере сгорания в начальный момент после воспламенения. Однако из-за увеличения характерного времени пребывания капель снижение температуры в процессе дальнейшего движения поршня происходит существенно медленнее. Снижение средней температуры в камере сгорания приводит к заметному снижению выбросов окислов азота (рис. 7в). Так, было показано, что увеличение диаметра сопла форсунки на 25 \% приводит к суммарному снижению выбросов NO на 35 \%. С другой стороны, уменьшение диаметра капель и улучшение перемешивания, происходящие при уменьшении сопла, приводят к существенному повышению эффективности процессов горения, что сказывается на недожоге и выбросах сажи. Так, было показано, что уменьшение диаметра сопла на 25 \% приводит к уменьшению образования сажи на 16 \% и уменьшению недожога топлива на $7 \%$.

\section{2. Исследование влияния угла раскрытия факела топливных форсунок}

Еще одной важной характеристикой работы топливной форсунки дизельного двигателя является угол раскрытия факела или угол конусности спрея. Глубина проникновения спрея вместе с углом его конусности определяют объем захвата аэрозольным облаком пространства камеры сгорания, что в конечном итоге определяет величину топливовоздушного соотношения внутри аэрозольного облака, от которой в значительной степени зависит эффективность процесса сгорания топлива. Угол конусности топливной форсунки варьировал в диапазоне от 10 до 20․ При этом остальные характеристики форсунки и двигателя оставались неизменными. Качественно влияние угла конусности форсунки на локальные характеристики

$$
-675-
$$




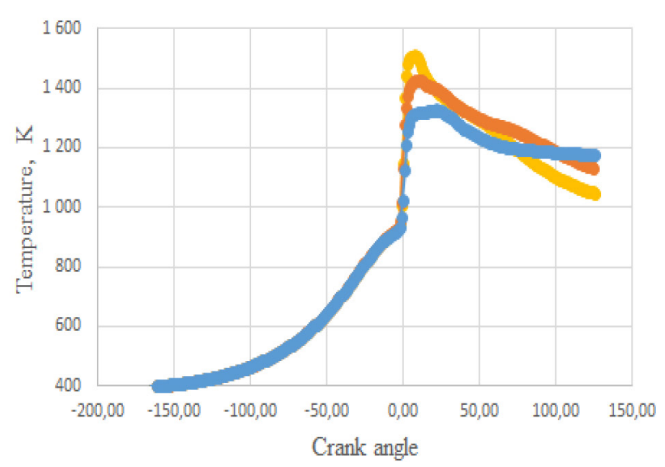

a)

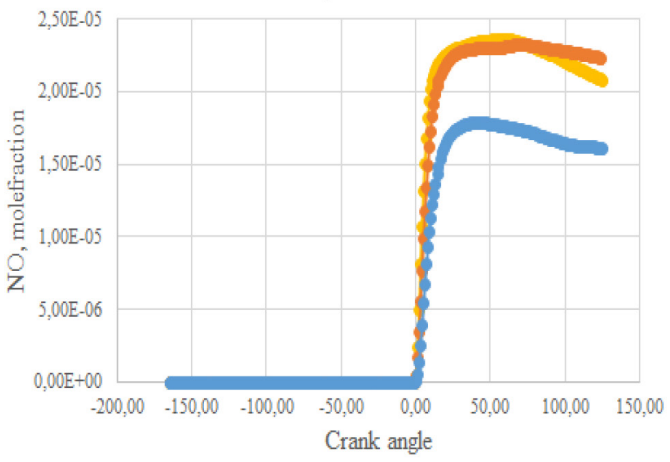

в)

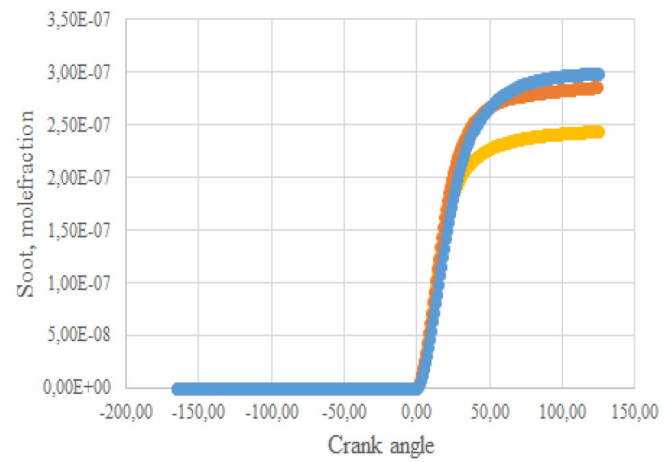

б)

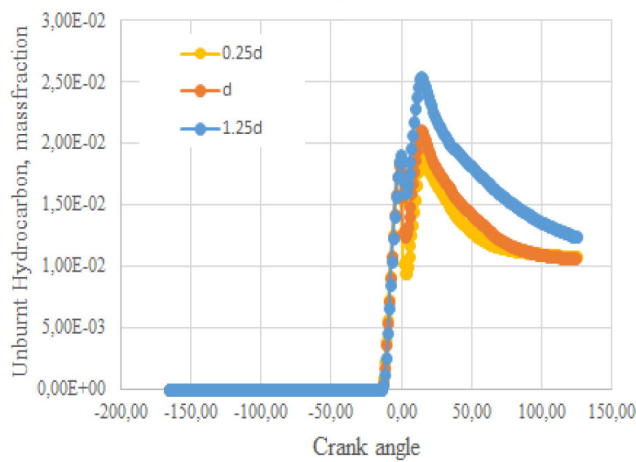

2)

Рис. 7. Средние значения температуры (a), объемной доли сажи (б), окислов азота (в) и недожога топлива (г) в камере сгорания в зависимости от диаметра сопла топливной форсунки

Fig. 7. Average values of temperature (a), volume fraction of soot $(\sigma)$, nitrogen oxides $(b)$ and underburnt hydrocarbon (d) in the combustion chamber at different fuel injector nozzle diameter

процессов перемешивания и горения дизельного топлива можно видеть на рис. 8,9 . Анализ результатов моделирования позволил выделить следующие закономерности влияния угла конусности. Угол раскрытия спрея значительно влияет на эффективность перемешивания топлива и воздуха. С увеличением угла раскрытия спрея увеличивается объем пространства камеры сгорания, в который попадают капли топлива, тем самым улучшается общее перемешивание. С уменьшением угла конусности топливная смесь локализуется в более узкой области пространства камеры сгорания, что ухудшает характеристики перемешивания. Топливовоздушное перемешивание пропорционально площади поперечного сечения факела и приблизительно пропорционально величине угла раскрытия конуса. С другой стороны, результаты моделирования показывают, что при слишком большом угле раскрытия спрея периферийная часть капель достигает стенок камеры сгорания, не успев испариться и воспламениться. В результате чего на стенках камеры сгорания формируется топливная пленка, скорость испарения которой существенно меньше, чем в каплях. Из-за этого возрастает недожог и количество сажи.

Количественно влияние угла конусности на характеристики процессов горения дизельного топлива показано на рис. 10. Как видим, угол конусности оказывает относительно слабое влияние на поведение средней температуры в камере сгорания. С увеличением угла конусно- 


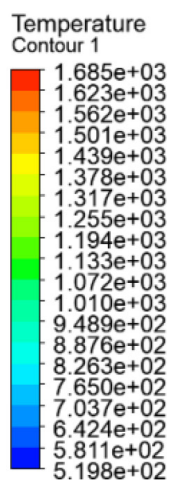

$[\mathrm{K}]$

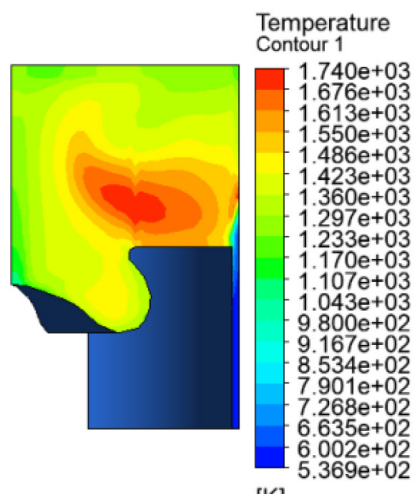

a)
[K]

б)

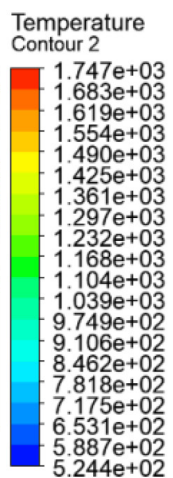

[K]

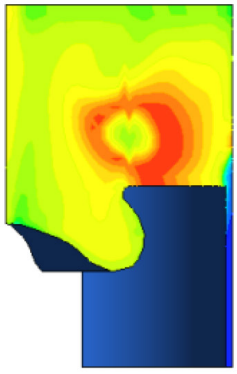

B)

Рис. 8. Температура в камере сгорания дизель-генератора при различных углах конусности спрея: $a-10^{\circ}$; $\sigma-15^{\circ} ; в-20^{\circ}$

Fig. 8. Temperature in the combustion chamber of a diesel generator at different spray angles: $a-10^{\circ}, \sigma-15^{\circ}$, b $-20^{\circ}$

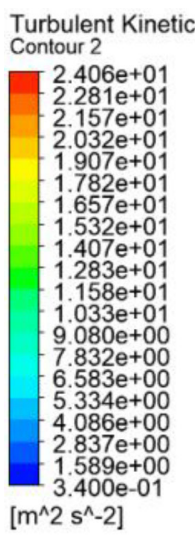

Turbulent Kinetic Energy

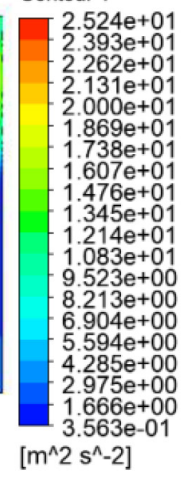

б)
Turbulent Kinetic Energy
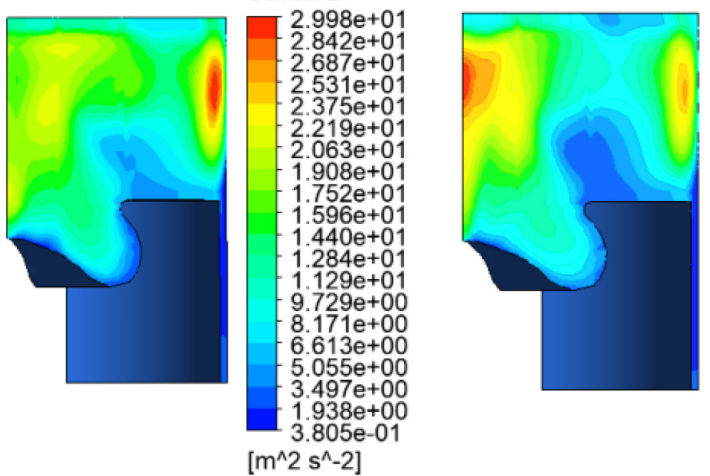

в)

Рис. 9. Турбулентная кинетическая энергия в камере сгорания дизель-генератора при различных углах конусности спрея: $a-10^{\circ} ; \sigma-15^{\circ} ; в-20^{\circ}$

Fig. 9. Turbulent kinetic energy in the combustion chamber of a diesel generator at different spray angles: $a-10^{\circ}$, $\sigma-15^{\circ}, 6-20^{\circ}$

сти средняя температура незначительно снижается. При больших углах конусности по обозначенным выше причинам на 20 \% возрастает недожог топлива и выбросы сажи.

\section{3. Исследование влияния угла опережения впрыска}

Важнейшей характеристикой, влияющей на экономические и экологические характеристики дизельного двигателя, является оптимальная настройка угла опережения впрыска. Двигатель будет иметь наилучшие мощностные и экономические показатели работы, если топливо максимально сгорает при нахождении поршня около верхней мертвой точки. Чтобы обеспечить выполнение этого требования, необходимо впрыскивать топливо с опережением, т. е. до прихода поршня в верхнюю мертвую точку. Величину опережения, выраженную в градусах

$$
-677-
$$




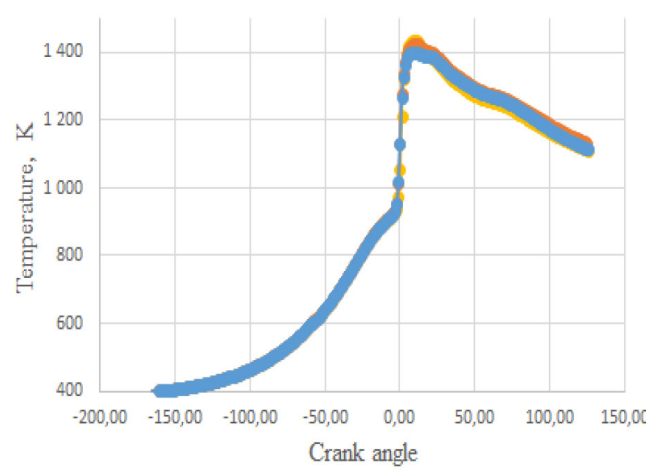

a)

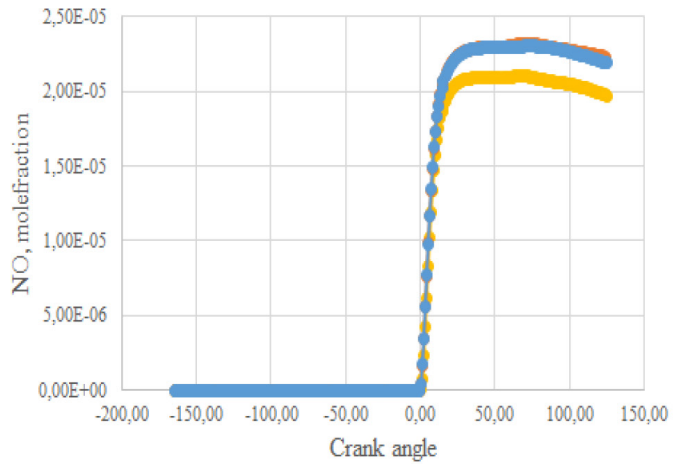

B)

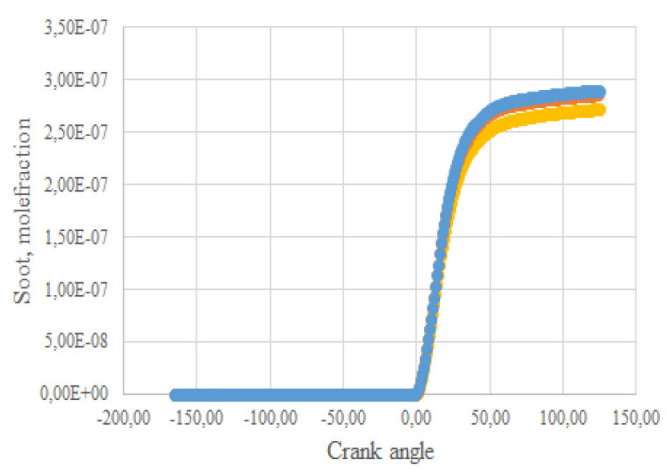

б)

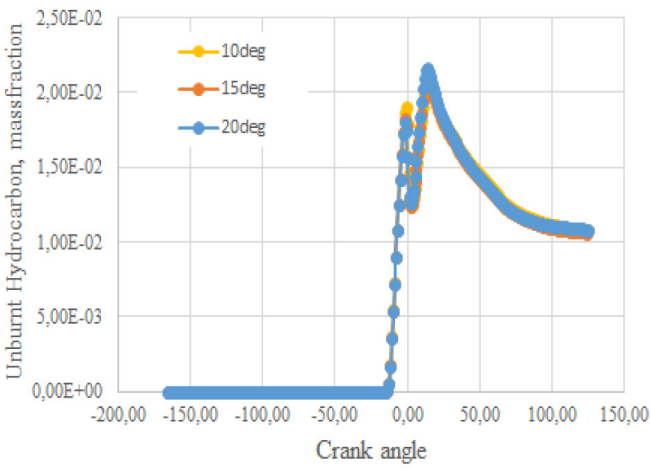

2)

Рис. 10. Средние значения температуры (a), объемной доли сажи (б), окислов азота (в) и недожега топлива (г) в камере сгорания в зависимости от раскрытия конуса распыла

Fig. 10. Average values of temperature $(a)$, volume fraction of soot $(\sigma)$, nitrogen oxides $(b)$ and underburnt hydrocarbon (2) in the combustion chamber at different fuel injector spray cone angles

угла поворота коленчатого вала, называют углом опережения впрыска $[15,16]$. В общем случае величина этого угла зависит от большого количества параметров (мощности двигателя, количества оборотов, качества топлива и др.). В данной работе проведено исследование влияния угла опережения зажигания при номинальных характеристиках двигателя. Величина угла опережения варьировалась в диапазоне от -25 до $-5^{\circ}$. Основные результаты моделирования приведены на рис. 11.

Анализ моделирования показывает, что при больших углах опережения топливо впрыскивается в камеру сгорания слишком рано, когда температура воздуха в цилиндре еще невысока. В этих условиях скорость испарения топлива очень низкая. Капли не успевают испариться и воспламениться, и большая их часть сепарируется на стенки камеры, ухудшая характеристики сгорания. Еще одним негативным фактором при раннем впрыске является рост давления в камере в процессе воспламенения топлива, который препятствует движению поршня в верхнюю мертвую точку. С увеличением угла опережения возрастают выбросы окислов азота и недожог топлива. В то же время выбросы сажи несколько снижаются. Исследование процессов тепломассообмена дизельного двигателя на режиме номинальной мощности при сохранении энергетических показателей выявило, что уменьшение угла опережения впрыска с $-25^{\circ}$ до $-5^{\circ}$ позволяет уменьшить содержания оксидов азота более чем в два раза за счет снижения макси- 


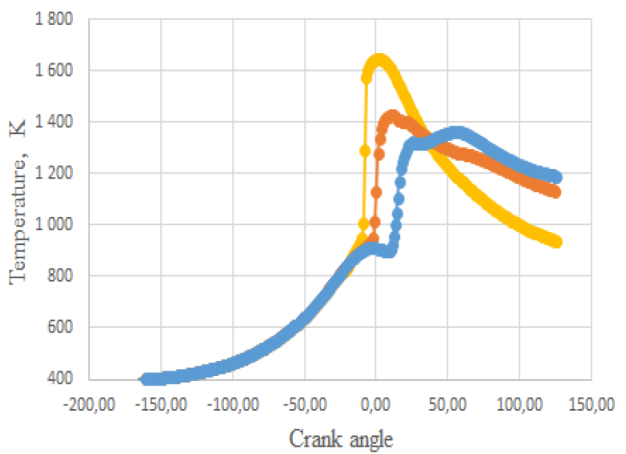

a)

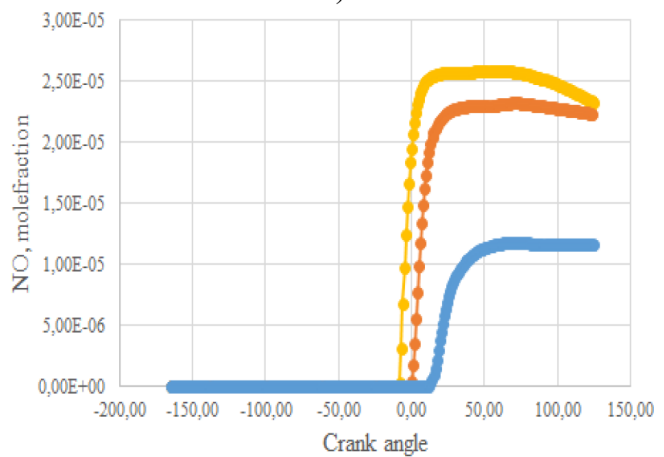

в)

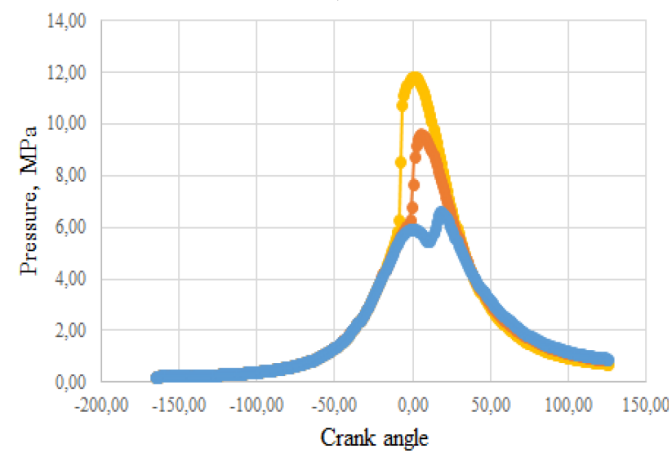

d)

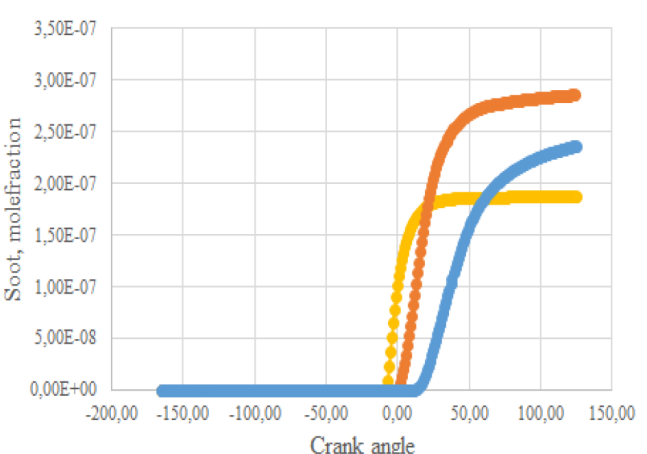

б)

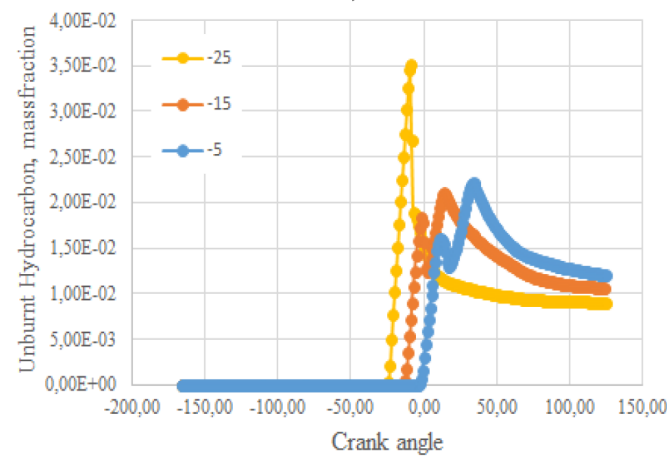

2)

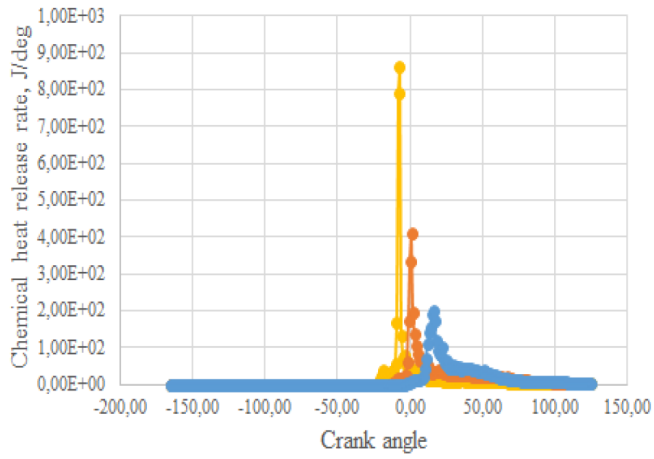

e)

Рис. 11. Средние значения температуры (a), объемной доли сажи (б), окислов азота (в), недожога топлива (2), давления (d) и скорость тепловыделения химической реакции (e) в камере сгорания в зависимости от угла опережения впрыска топлива

Fig. 11. Average values of temperature (a), volume fraction of soot ( 6$)$, nitrogen oxides (b), underburnt hydrocarbon (2), pressure $(\partial)$ and chemical heat release rate $(e)$ in the combustion chamber at the fuel injection advance angle

мальной температуры в камере сгорания. Кроме того, позволяет уменьшить скорость нарастания давления за счет сокращения периода задержки воспламенения и увеличения продолжительности сгорания.

С другой стороны, при слишком малых углах опережения впрыска характеристики дизельного двигателя также ухудшаются. Топливо в этом случае сгорает при такте расширения. Скорость его сгорания снижается, а поверхность соприкосновения горячих газов со стенками 
цилиндра увеличивается. В этом случае много тепла будет отдано в охлаждающую воду и выброшено с отработавшими газами $[15,16]$.

Ранний предварительный впрыск топлива положительно влияет на шумность работы двигателя. При слишком позднем впрыске топлива также существенно возрастает его недожог. В результате чего снижается КПД, увеличивается закоксованность двигателя и сокращается интервал замены масла. Результаты моделирования показали, что для данного двигателя в номинальном режиме работы угол опережения впрыска $-15^{\circ}$ следует считать оптимальным.

\section{4. Исследование влияния угла наклона оси форсунки}

в камере сгорания

Еще одним фактором, которым можно управлять характеристиками сгорания дизельного двигателя, является угол наклона оси форсунки. Рассмотрено два дополнительных угла отклонения оси форсунки от номинального значения. Результаты моделирования этих вариантов приведены на рис. 12,13 . Было показано, что управление углом позиционирования форсунки также значительно влияет на аэродинамику потоков в цилиндре дизельного двигателя. Это, в свою очередь, в значительной мере сказывается на характеристиках перемешивания и режиме испарения топлива, влияя на экологические и энергетические характеристики всего двигателя.

Из анализа данных, приведенных на рис. 13, следует, что изменение угла наклона оси топливного спрея в пределах $10^{\circ}$ по высоте приводит к уменьшению выбросов окислов азота на $60 \%$ и уменьшению выбросов сажи на $9 \%$. Но при этом на $40 \%$ возрастает недожог топлива. Исходя из этого угол наклона оси форсунки, равный $110^{\circ}$, можно рассматривать как оптимальный.

\section{Заключение}

Разработана математическая модель процессов тепломассообмена в камере сгорания дизель-генераторных установок с вентильно-индукторными генераторами. Модель учитывает реальную геометрию камеры сгорания и условия работы дизельного двигателя, характе-

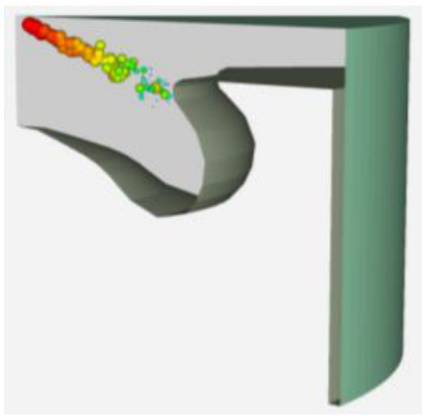

a)

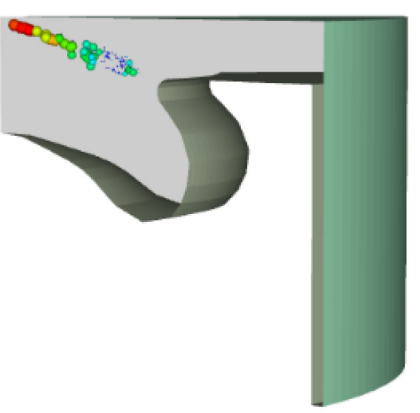

б)

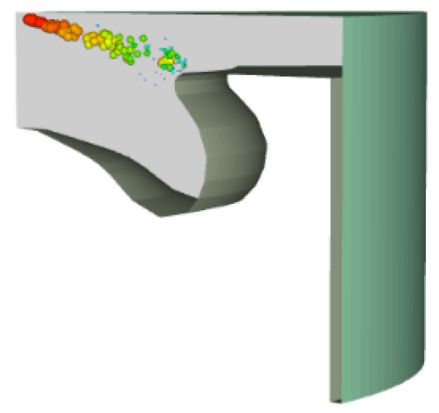

в)

Рис. 12. Характеристики распыла дизельного топлива в камере сгорания при различных углах наклона форсунки: $\mathrm{a}--5^{\circ} ; \sigma-0^{\circ} ;$ в $-+5^{\circ}$

Fig. 12. Characteristics of diesel fuel spray in the combustion chamber at different nozzle angles: a) $-5^{\circ}$, b) $0^{\circ}$, c) $+5^{\circ}$ 


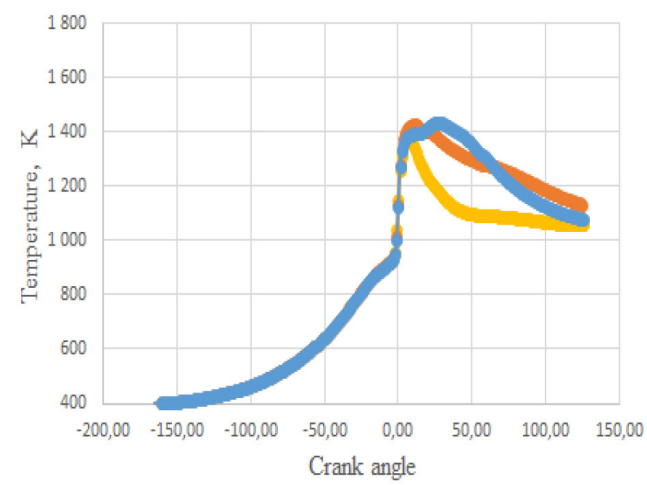

a)

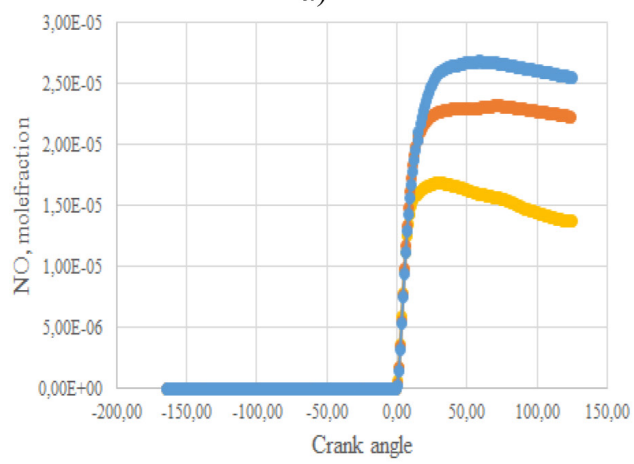

в)

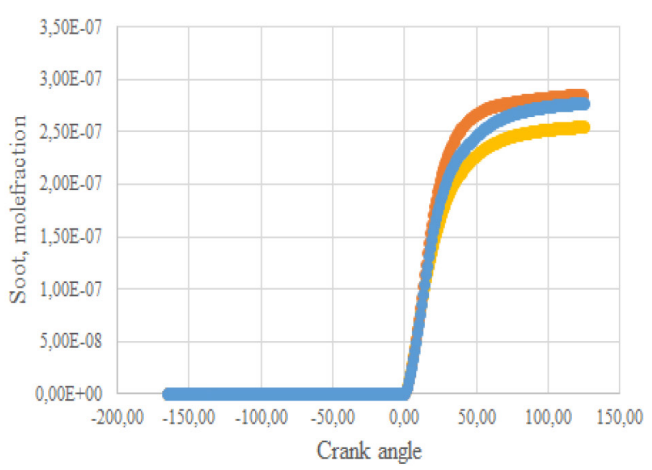

б)

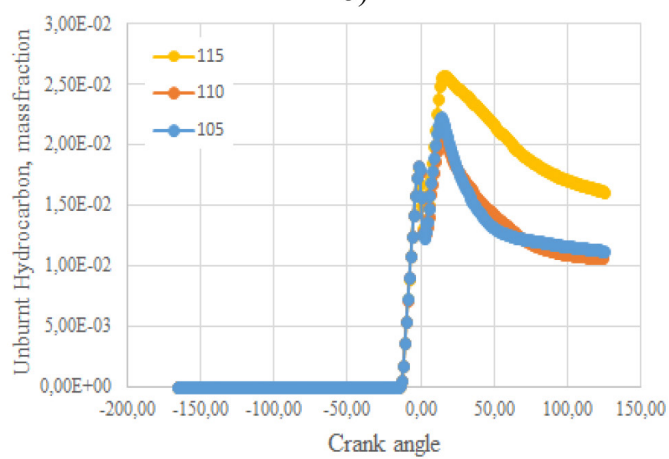

2)

Рис. 13. Средние значения температуры (a), объемной доли сажи (б), окислов азота (в) и недожога топлива (2) в камере сгорания в зависимости от угла направления форсунки

Fig. 13. Average values of temperature (a), volume fraction of soot (б), nitrogen oxides $(8)$ and underburnt hydrocarbon (2) in the combustion chamber at angle of direction of the nozzle

ристики работы форсунок и свойства дизельного топлива. С помощью разработанной модели проведено систематическое расчетное исследование по оптимизации работы топливных форсунок и характеристик их распыла для дизель-генератора. Исследовано влияние диаметра сопла форсунки, угла ее наклона, угла конусности спрея и угла опережения впрыска на локальные и интегральные характеристики работы дизель-генератора. Особое внимание было уделено влиянию характеристик распыла топлива на выбросы в процессе работы дизель-генератора.

Установлено, что уменьшение угла опережения впрыска с $-25^{\circ}$ до $-5^{\circ}$ позволяет уменьшить содержания оксидов азота более чем в два раза за счет снижения максимальной температуры в камере сгорания, а также на 20 \% уменьшить выбросы сажи. Однако при этом в 1,2 раза возрастает недожог. Таким образом, результаты моделирования показали, что для рассматриваемой модели двигателя в номинальном режиме работы угол опережения впрыска $-15^{\circ}$ следует считать оптимальным.

Показано, что увеличение диаметра сопла форсунки на 25 \% приводит к суммарному снижению выбросов NO на 35 \%. С другой стороны, уменьшение диаметра капель и улучшение перемешивания, происходящие при уменьшении сопла, приводит к существенному повышению эффективности процессов горения, что сказывается на недожоге и выбросах сажи. Так, 
было продемонстрировано, что уменьшение диаметра сопла на 25 \% приводит к уменьшению образования сажи на 16 \% и уменьшению недожога топлива на 7 \%.

Выявлено, что с увеличением угла конусности спрея происходит увеличение недожога и выбросов сажи за счет наброса капель на стенки камеры сгорания.

Установлено, что изменение угла наклона оси топливного спрея в пределах $10^{\circ}$ по высоте приводит к уменьшению выбросов окислов азота на 60 \% и уменьшению выбросов сажи на $9 \%$. Но при этом на $40 \%$ возрастает недожог топлива. Исходя из этого угол наклона оси форсунки, равный $110^{\circ}$, можно рассматривать как оптимальный.

Таким образом, было показано, что при помощи даже незначительной оптимизации характеристик работы топливной форсунки можно в значительной мере добиться снижения вредных выбросов в процессе работы дизель-генератора.

\section{Благодарности / Acknowledgements}

Исследование выполнено при финансовой поддержке РФФИ, Правительства Красноярского края, Краевого фонда науки и ООО «Нижнебогучанская ГЭС» в рамках научного проекта № 20-48-242909 «Теоретические основы построения адаптивных регуляторов частоты вращения и качества генерируемой электроэнергии дизель-генераторных установок с вентильноиндукторными генераторами».

The research was carried out with the financial support of the RFBR, the Government of the Krasnoyarsk Territory, the Regional Science Foundation and Nizhneboguchanskaya HPP LLC within the framework of the scientific project No. 20-48-242909 «Theoretical foundations for the construction of adaptive speed regulators and the quality of generated electricity for diesel generator sets with valve-inductor generators».

\section{Список литературы / References}

[1] Орлов А.В., Путятинский В.А., Сапожников В.В. Перспективы создания дизельэлектрических установок с переменной частотой вращения, Судостроение, 1976, 10, 28-29 [Orlov A. V., Putyatinskiy V. A., Sapozhnikov V.V. Prospects for the creation of variable speed diesel electric installations, Shipbuilding, 1976, 10, 28-29 (in Russian)]

[2] Крутов В.И. Автоматическое регулирование двигателей внутреннего сгорания, М.: Машиностроение, 1979. 615 с. [Krutov V. I. Automatic regulation of internal combustion engines: a manual for technical colleges, Moscow, Mashinostroyeniye, 1979, 615 p. (in Russian)]

[3] Хватов О.С. и др. Высокоэффективная дизель-генераторная электростанция переменной частоты вращения на основе машины двойного питания, Приводная техника, 2010, 5, 14-19. [Khvatov O.S. and others. Highly efficient diesel-generator power plant of variable speed based on a dual-feed machine, Drive Technology, 2010, 5, 14-19 (in Russian)]

[4] Поляков И.С. Дизель-генераторная установка переменной частоты вращения, дисс. ... канд. техн. наук. Нижний Новгород, 2013, 155 с. [Polyakov I. S. Diesel generator set of variable rotation frequency, diss. Cand. tech. sciences. Nizhny Novgorod 2013, 155 p. (in Russian)]

[5] Гузей Д.В., Минаков А.В., Пантелеев В.И.,. Пряжников М.И, Платонов Д.В., Жигарев В.А. Математическая модель и расчетное исследование процессов тепломассообмена в камере сгорания дизель-генераторных установок с вентильно-индукторным генератором. 
Журнал Сибирского федерального университета. Техника и технология, 2020, 13(5), 611-625. [Guzei D. V., Minakov A. V., Panteleev V.I., Pryazhnikov M. I., Platonov D. V., Zhigarev V.A. The Mathematical Model and Numerical Study of Heat and Mass Transfer Processes in the Combustion Chamber of Diesel-Generator Units with a Valve-Inductor Generator J. Sib. Fed. Univ. Eng. technol., 2020, 13(5), 611-625 (in Russian)]

[6] Desantes, J. M., García-Oliver, J. M., Novella, R., \& Pachano, L. A numerical study of the effect of nozzle diameter on diesel combustion ignition and flame stabilization. International Journal of Engine Research, 2019, 21(1), 101-121.

[7] Singh, A. P., Shukla, P. C., Hwang, J., \& Agarwal, A. K. (Eds.). Simulations and Optical Diagnostics for Internal Combustion Engines. Springer, Singapore, 2020, 163 p.

[8] Shervani-Tabar, M. T., Sheykhvazayefi, M., \& Ghorbani, M. Numerical study on the effect of the injection pressure on spray penetration length. Applied Mathematical Modelling, 2013, 37(14-15), $7778-7788$.

[9] Han Z., Reitz R.D. Turbulence modeling of internal combustion engines using RNG $\kappa-\varepsilon$ models. Combust. Sci. Technol., 1995, 106(4-6), 267-295.

[10] Sun Y., Reitz R.D. Modeling Low-Pressure Injections in Diesel HCCI Engines, ILASS Americas, 20th Annual Conference on Liquid Atomization and Spray Systems, Chicago, 2007.

[11] Ra Y., Reitz R.D. A vaporization model for discrete multi-component fuel sprays, Int. J. Multiphase Flow, 2009, 35, 101-117.

[12] Kong S.-C., Reitz, R.D. Use of Detailed Chemical Kinetics to Study HCCI Engine Combustion With Consideration of Turbulent Mixing Effects, Trans. Am. Soc. Mech. Eng., 2002, 124, 702-707.

[13] Hiroyasu H. and Kadota T., Models for Combustion and Formation of Nitric Oxide and Soot in DI Diesel Engines, SAE Technical Paper 760129, SAE Technical Paper Series, 1976.

[14] Nagle J., Strickland-Constable R.F. Oxidation of Carbon Between 1000-2000 ${ }^{\circ} \mathrm{C}$, Proceedings of the Fifth Carbon Conference, Vol. 1, New York: Pergamon, 1962, 154-164 p.

[15] Алексеев В.П., Воронин В.Ф. и др. Двигатели внутреннего сгорания: Устройство и работа пориневых и комбинированных двигателей. М.: Машиностроение, 2010. 288 с. [Alekseev V.P., Voronin V.F. et al. Internal combustion engines: Design and operation of piston and combined engines. Moscow: Mashinostroenie, 2010. 288 p. (in Russian)]

[16] Гусаков С.В. Перспективы применения в дизелях альтернативных топлив из возобновляемых источников. М.: РУДН, 2008. 288 с [Gusakov S. V. Prospects for the use of alternative fuels from renewable sources in diesel engines. M.: RUDN, 2008. 288 p. (in Russian)] 